\title{
Time-resolved 3D-CMR using free-breathing 2D-acquisitions
}

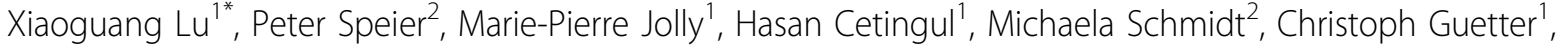 \\ Carmel Hayes ${ }^{2}$, Arne Littmann², Qiu Wang ${ }^{1}$, Mariappan S Nadar ${ }^{1}$, Frank Sauer ${ }^{1}$, Edgar Mueller ${ }^{2}$ \\ From 17th Annual SCMR Scientific Sessions \\ New Orleans, LA, USA. 16-19 January 2014
}

\section{Background}

A typical CMR exam consists of a limited number of 2D scans that provide standard views of the heart. Diagnosis is limited to these select views. For the acquisition, multiple breath-holds are required - a challenge for many patients. As an improvement, we have investigated a free-breathing (FB) 2D acquisition protocol in conjunction with a novel reconstruction approach. The method provides $3 \mathrm{D}+$ time cine data with full heart coverage while simplifying the acquisition.

\section{Methods}

FB images were acquired with a fluoroscopic radial bSSFP, image rate $40-50 \mathrm{~ms}$, res. $(1.82 \times 1.82 \times 7) \mathrm{mm}^{3}$. ECG time-after-trigger was recorded for retrospective mapping to cardiac phases. Volume coverage was achieved by changing the scan plane position or orientation between images slightly in order to not disturb the spin steady state. Images were reconstructed offline with a Compressed Sensing algorithm based on [Liu J et.al., ISMRM, Melbourne, Australia, 2012, P.4249]. Two datasets with approximately orthogonal scan planes were acquired: a short-axis (SX) set covering the heart with steps of $0.05 \mathrm{~mm}$, and a long-axis (LX) set, rotating $180^{\circ}$ around the left-ventricular long axis in steps of $0.1^{\circ}$. Our reconstruction pipeline consists of five modules. Modules b)-d) are used to reconstruct a volume at one cardiac phase. The pipeline is described for reconstructing SX data using LX anchors. Modules: a) For each acquired cardiac cycle, the cardiac phase is normalized to a $30 \mathrm{~ms}$ time step by deformation-field-assisted interpolation. b) Two approximately orthogonal LX slices, with most consistent SX-LX data correlation at the intersection lines (CAI) over all cardiac phases, are selected as anchors. c) CAI analysis computes the correlation along the intersection line between LX anchors and SX slices, and selects SX candidates with high correlation that actually correspond to SX slices on a similar respiratory phase. d) Residual misalignments between selected SX slices are iteratively corrected by optimizing the correlation with LX anchors while being constrained by neighboring SX slices through non-rigid registration. e) Standard scattered interpolation is applied to interpolate voxel intensities on a regular $1.82^{3} \mathrm{~mm}^{3} 3 \mathrm{D}$ grid.

\section{Results}

The proposed automatic pipeline as a prototype was implemented in Matlab [Mathworks, Inc., MA, USA] and successfully applied to four volunteer data sets to generate 3D+time data on a $1.82^{3} \mathrm{~mm}^{3}$ grid based on SX data with LX anchors. The average distance between contributing SX slices was $\sim 3.14 \mathrm{~mm}$ (see Figure 2).

\section{Conclusions}

We demonstrated the feasibility of fully automated reconstruction of 3D CINEs from orthogonal 2D-FB acquisitions. Further investigations to characterize the performance and robustness of the method are underway. 


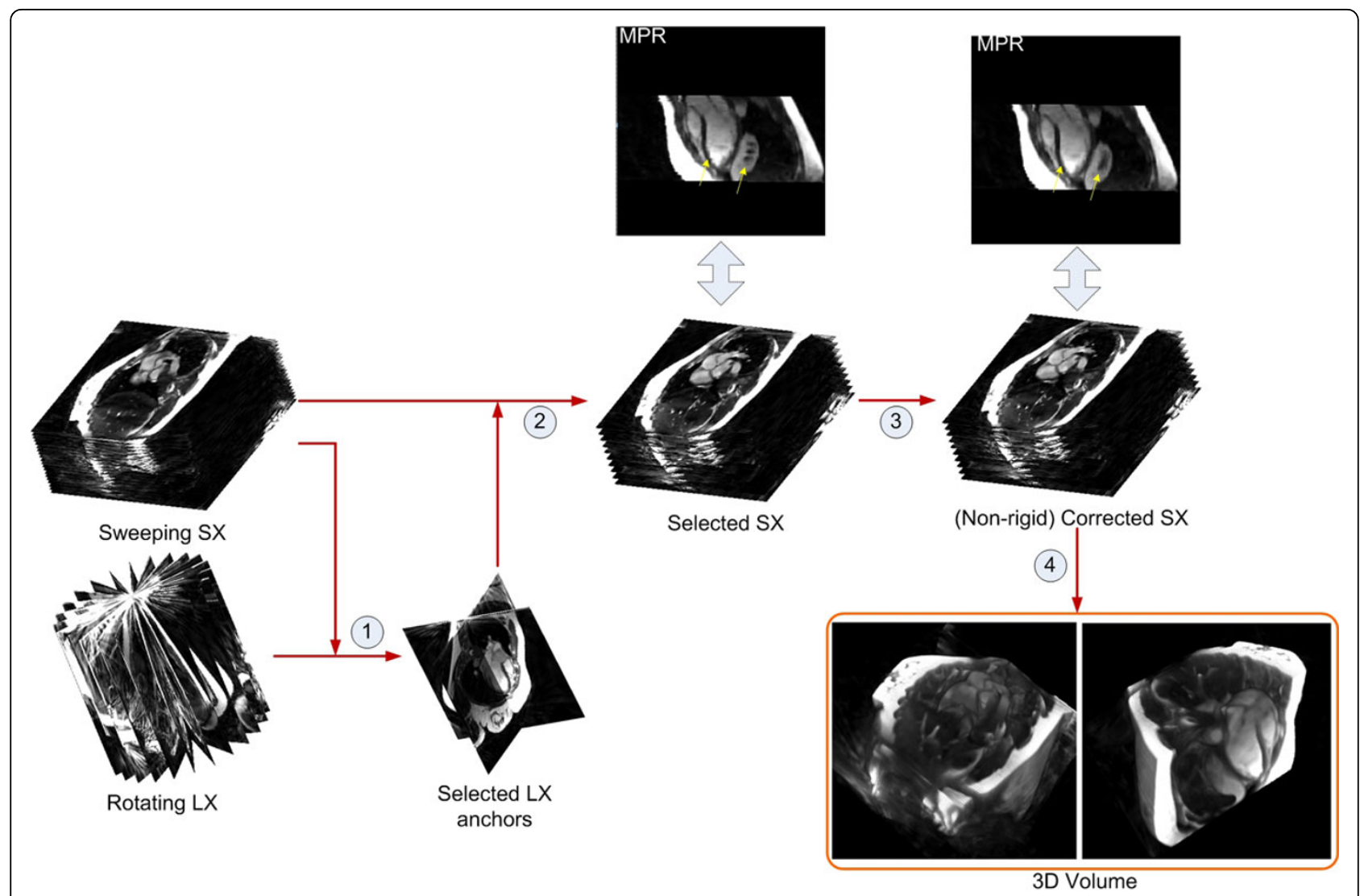

Figure 1 Volume reconstruction at one cardiac phase with four major steps: 1. Anchor selection; 2 . Slice selection with anchors; 3. Non-rigid slice correction; 4. Scattered interpolation.
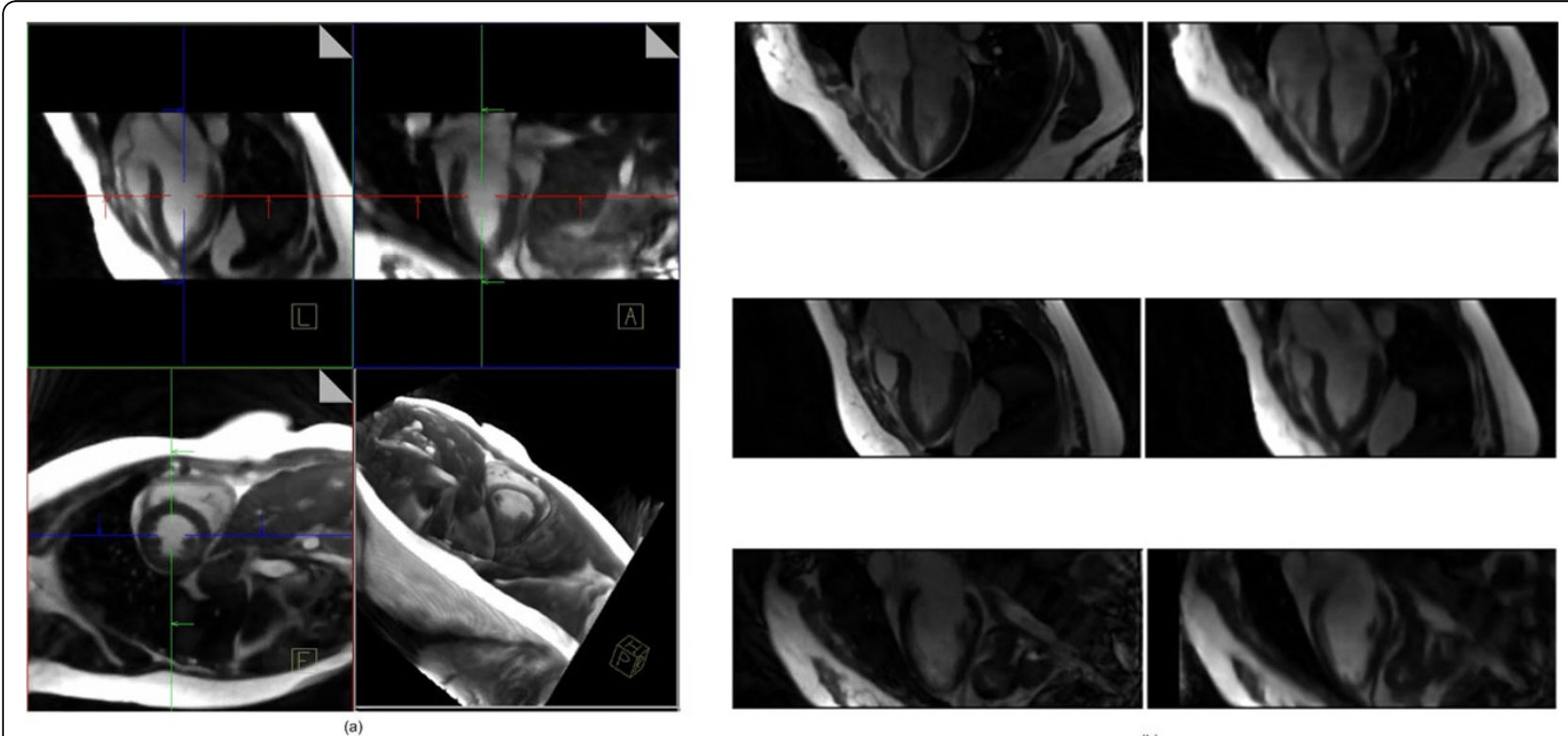

(b)

Figure 2 Example of reconstruction results: a) three orthogonal MPRs and a 3D rendering; b) three MPRs from reconstructed volumes (right) compared with acquired LX images at the same views (left). 


\section{Funding}

This work is sponsored by Siemens.

\section{Authors' details}

${ }^{1}$ Imaging and Computer Vision, Siemens Corporate Research, Princeton, New Jersey, USA. ${ }^{2}$ MR Application \& Workflow Development, Siemens AG,

Erlangen, Germany.

Published: 16 January 2014

doi:10.1186/1532-429X-16-S1-P50

Cite this article as: Lu et al.: Time-resolved 3D-CMR using free-breathing

2D-acquisitions. Journal of Cardiovascular Magnetic Resonance 201416

(Suppl 1):P50.

Submit your next manuscript to BioMed Central and take full advantage of:

- Convenient online submission

- Thorough peer review

- No space constraints or color figure charges

- Immediate publication on acceptance

- Inclusion in PubMed, CAS, Scopus and Google Scholar

- Research which is freely available for redistribution 